\title{
Floor Map Visualizations of Medical Volume Data
}

\author{
Nico Merten \\ Sylvia Saalfeld \\ Bernhard Preim \\ $1^{\text {st }}$ Affiliation: Department of Simulation and Graphics, Otto-von-Guericke University \\ $2^{\text {nd }}$ Affiliation: Research Campus STIMULATE \\ Universitätsplatz 2 \\ D-39106, Magdeburg, Germany \\ [nmerten, sylvia, preim]@isg.cs.uni-magdeburg.de
}

\begin{abstract}
Typically, volumetric medical image data is examined by assessing each slice of an image stack individually. However, this enables observers to assess in-plane spatial relationships between anatomical structures only and requires them to keep track of relationships along the third anatomical plane mentally. Therefore, visualization techniques are researched to support this task by depicting spatial information along the third plane, but they can introduce a high degree of abstraction. To overcome this, we present a novel approach that transforms image stacks with labeled anatomical structures into maps with a three-dimensional layout, namely floor maps. Since this approach increases the visual complexity under certain conditions, some clinical application scenarios, e.g. diagnosis and therapy planning, probably will not benefit. Thus, the approach is mainly aimed to support student training and the generation of clinical reports. We also discuss how to enhance the slice-based exploration of medical image stacks via floor maps and present the results of an informal evaluation with three trained anatomists.
\end{abstract}

\section{Keywords}

Floor Maps, Exploration Support for Medical Volume Data, Abstraction

\section{INTRODUCTION \& MOTIVATION}

Over the last decades, much research was carried out to improve medical image scanners, such as Computed Tomography (CT) or Magnetic Resonance Imaging (MRI) scanners, e.g. with respect to spatial resolution. Thus, less anatomical information is compiled and mapped into individual Volume Elements (voxels). On the one hand, this improved the versatility and fidelity of the image data. On the other hand, this results in more visual information that has to be assessed. Generally, this is achieved by axial slicing, e. g. via computer mouse scrolling. The main disadvantage is that users have to understand three-dimensional spatial relationships between anatomical structures although only in-plane relationships are depicted. Especially for medical students in training this can become mentally exhausting, because they are not yet accustomed to these tasks.

The main contribution of this work is a processing pipeline that can transform labeled, medical image stacks into interactively explorable floor maps. These maps are a well-known concept to provide spatial in-

\footnotetext{
Permission to make digital or hard copies of all or part of this work for personal or classroom use is granted without fee provided that copies are not made or distributed for profit or commercial advantage and that copies bear this notice and the full citation on the first page. To copy otherwise, or republish, to post on servers or to redistribute to lists, requires prior specific permission and/or a fee.
}

formation in large, multi-story buildings, such as malls, hospitals, or federal buildings [11]. These maps aim to use the natural exploration-supporting properties of maps while introducing only a moderate degree of abstraction. Many people are used to maps, e.g. from various handheld devices. Additionally, maps support our visuo-spatial working memory, which is a mental resource that we require for orientation and navigation tasks in spatial environments. Furthermore, since CT or MRI scans are multiple, stacked images, their data layout is three-dimensional and the visual layout of maps can be three-dimensional, too. Finally, maps and medical image visualization techniques purposefully abstract and simplify geometric details. This can be beneficial for anatomical education and report generation, since, in the beginning, understanding spatial relationships between anatomical structures is more important than learning geometric details, and the generation of easy-to-understand and interpret documentations of findings is an important task in every clinical workflow. Therefore, we hypothesize that floor maps are suitable to offer exploration support for medical image data.

\section{RELATED WORK}

One downside of the slice-based exploration and assessment of image stacks is that only the in-plane spatial relationships between structures are depicted. Thus, physicians have to keep track of spatial relationships along the third anatomical plane mentally. To support this task, $2 \mathrm{D}$ and $3 \mathrm{D}$ visualization techniques were developed that, 

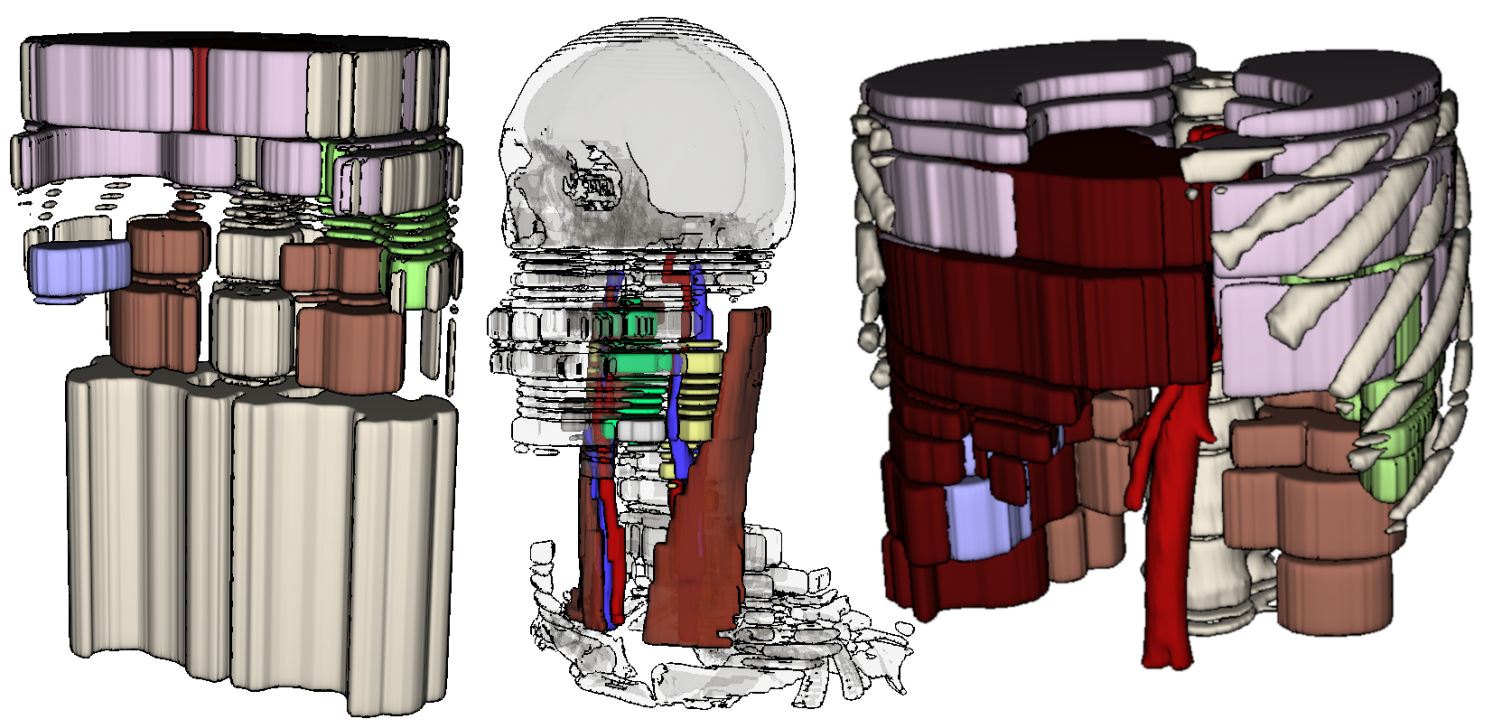

Figure 1: Floor map visualizations depicting three image data sets (cf. Tab. 1). Left (DS1): A standard floor map is depicted. Middle (DS2): Various structures, such as the two cervical muscles (brown), the arteries (red), and veins (blue), were re-merged. Right (DS3): The aorta and lateral ribs were re-merged while also preserving their shape.

in general, depict additional spatial information of the explored and assessed image stack along the third plane.

Exploration Support. Tietjen et al. [17] presented a 2D technique called lift charts: While an image stack is explored in a 2D view, the spatial extent of labeled structures along the third plane is depicted in an additional 2D chart. The result is an adapted bar chart with multiple vertical bars that can be interpreted as lifts that make it possible to distinct vertical sections. Generally,

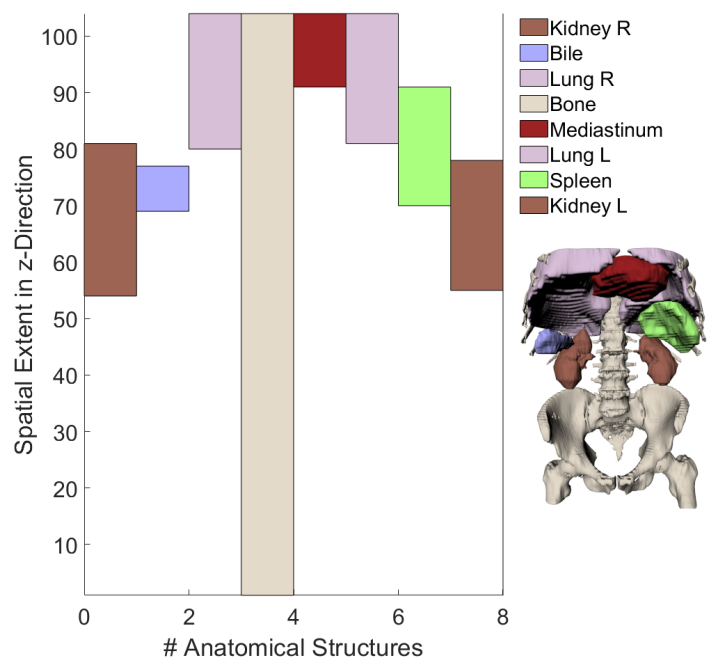

Figure 2: A lift chart to support the slice-based exploration of medical volume data as introduced by Tietjen et al. [17]. All labeled anatomical structures are represented via bars and their vertical positions and extents are defined by the $z_{\min }$ and $z_{\max }$ coordinates of their respective structure's Axis-Aligned Bounding Box. The respective data set is presented on the right. lifts are defined along the $\mathrm{z}$-axis, because tomographic scans are acquired and assessed in axial slices. However, lift charts can be applied for arbitrary projection planes. Furthermore, a unique color-coding is assigned to each structure type, such as bones and lymph nodes. The lift chart in Figure 2 was generated using Data Set 1 (see Tab. 1). The respective floor map can be seen in the leftmost subfigure of Figure 1.

Later, Balabanian et al. [3] used lift charts for their hierarchical graph network, which enabled them to use lift charts for anatomical substructures, too. Thus, there now exist multiple charts for one data set: Depending on the currently observed hierarchy level, a chart presents spatial relationships between structures and substructures at different scales. Diepenbrock et al. [7] extended lift charts to present information from scans of different modalities: Rather than using morphological scans only, their lift charts also present information from functional MRI (fMRI) scans like a graph plot.

Although lift charts are a straightforward visualization technique to enhance the slice-based exploration of image stacks, their overall degree of abstraction is very high. Following the theory of Viola and Isenberg [19], lift charts would rank high on the geometric and photometric abstraction axes, because only two values and one color are presented per structure. Estimations of the individual degrees of abstraction for lift charts and floor maps are depicted in Figure 3. When considering real-world anatomy as the starting point and biochemical processes at the end point of the scale axis, lift charts and floor maps are located at a very low point, because they are used for high-level morphological information. Moreover, since only one scan is used, there is no temporal information; thus, both techniques introduce no 
temporal abstraction. Techniques with a low photometric abstraction use complex light propagation models, whereas more photometrically abstract techniques use simpler shading or stylizations, such as flat shading or line drawings. When techniques introduce a low degree of geometric abstraction, many shape details are preserved, whereas objects will get very simplified when a high degree of geometric abstraction is used. The degrees of photometric and geometric abstraction are very high for lift charts, since only their vertical extent is preserved and presented in a simple color-coding. Thus, we hypothesized that floor maps are more appropriate to offer exploration support for medical volume data, because they preserve more geometric details, such as the axial shape of structures, and present them using ambient and diffuse shading, which further emphasize valleys between floors and rooms.

Mindek et al. [14] presented a different type of exploration support by virtually altering the slicing speed during 2D exploration via non-linear interaction. They define representative slices if chosen structures undergo large morphological changes between adjacent slices, for example, if the cross-section areas of blood vessels change or if they branch. During 2D exploration, only these representative slices are presented to the user, which results in a slower exploration in regions with large changes, because there are more representative slices. Similarly, in image regions with many morphological changes our method generates more floors and rooms. Consequently, both methods generate only few representative slices or large floors with few rooms in image regions with only small or no changes.

Digital 3D Maps. Research for digital maps focuses on

- pathway planning in emergency situations [6],

- generation of 3D models from 2D drawings [20],

- level-of-detail techniques for indoor maps [13], or

- the ontological description of buildings [12].

Floor maps help users to familiarize with complex spatial layouts, such as offices, high-rise buildings, and

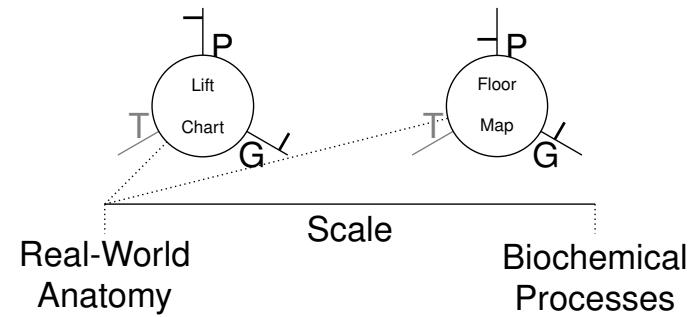

Figure 3: Ranking the lift chart and floor map visualization techniques using the theory of Viola and Isenberg [19]. The radiant axes show the Photometric, Geometric, and Temporal abstraction categories. Axes ticks show the estimated degrees of abstraction in the respective category. The T category is not applicable. malls [11]. To exploit this potential and to prevent visual clutter, special care is necessary. For example, color scales with only few colors that can be easily distinguished have to be defined and occlusion problems have to be controlled, e. g. by generating good default views on building models. Our approach enables users to interactively adapt the color-coding and opacity of structures.

\section{FLOOR MAP GENERATION}

In this section the data sets and processing pipeline to create interactive 3D floor map visualizations from medical image volumes are described. Figure 4 depicts the proposed pipeline to transform segmentation masks into an interactively explorable floor map visualization. After presenting the conceptual design, each step of the pipeline is described in Section 3.3.

\subsection{Image Data and Implementation}

To develop the proposed method, three CT scans were used (see Tab. 1). The processing pipeline, which transforms label images into floors and rooms, was implemented in MeVisLab 2.8.2 [15] (see Fig. and Sec. 4). Subsequently, users can use a Graphical User Interface (GUI) (see Fig. 9) to simultaneously explore the unaltered image stacks and floor maps.

\subsection{Conceptual Design}

When applying the floor map concept on medical volume data, we discussed how human anatomy can be transformed into floors and rooms. Generally, larger structures, such as organs, have a deformed, spherical shape with soft edges. There are some exceptions, such as extremity bones or intestines, which, while also having a round, have a rather elongated shape. Smaller structures, such as blood vessels, are tubular with circular or ellipsoid shaped cross-sections.

In contrast, buildings are man-made structures with considerable regularity. For most buildings, the ground plan is extruded vertically and divided horizontally into equally high floors. Thus, they often have a cuboid surface. However, especially for smaller buildings, such as homes, the inner floor layout can be very individual with varying room sizes, whereas for taller buildings, the floor layout can be very repetitive. Additionally, pathways, such as corridors, staircases and elevators, are straight, horizontally or vertically oriented tubes.

In clinical practice, physicians typically assess image stacks via axial slicing. Therefore, the proposed approach divides image stacks along the $\mathrm{z}$-axis to create floors, and to make them clearly visually distinguishable, small gaps are inserted between adjacent floors. However, although the gap size is adjustable, each division results in a geometric distortion. Therefore, the number of divisions should be minimal. 


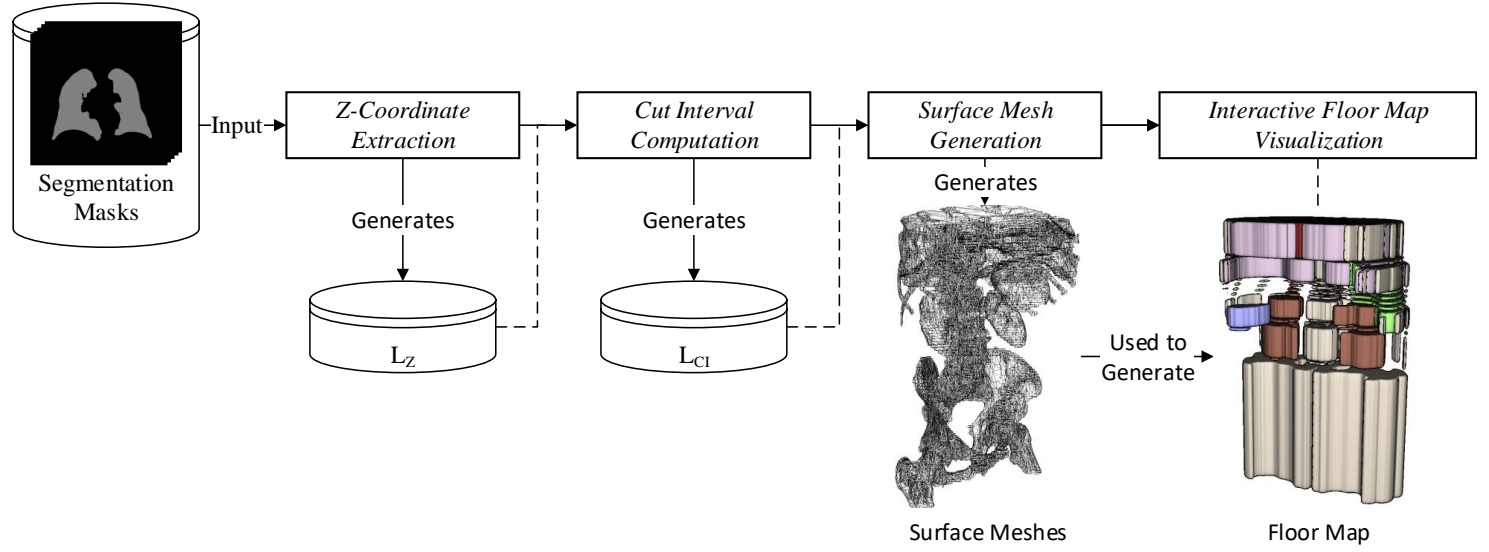

Figure 4: Pipeline to generate floor map visualizations from medical image stacks. $L_{Z}$ is a compiled list of unique $z_{\min }$ and $z_{\max }$ coordinates of all segmentation masks. $L_{C I}$ is a list of cut intervals that are used to divide the segmentation masks into floors.

To create building-like maps, rooms have to be created. Anatomical structures can have complex spatial arrangements, e.g. the heart is encompassed by the lungs, but they are clearly separated. This feature is considered in the processing pipeline by creating floors and rooms that are free from overlaps (cf. Figures 6 and 7). However, to enable an easy visual grouping of rooms that represent similar anatomical structures, they are color-coded identically. For example, in Figure 4 the kidneys and lungs are colored brown and pink, respectively.

\subsection{Image Stack Processing}

Z-Coordinate Extraction. In the first step of the processing pipeline, the $z_{\min }$ and $z_{\max }$ coordinates of all labeled structures' axis-aligned bounding boxes are extracted and compiled into a list named $L_{Z}$. Before the coordinates are compiled, certain structures can be marked as being too small for processing, if their spatial extent in $\mathrm{z}$-direction $\left(z_{\max }-z_{\min }\right)$ is below a user-defined threshold. Thus, they will not be divided into floors and their shape will be preserved. However, due to gaps between floors, they have to be moved to their correct vertical position for the final visualization. In Figure 5, lymph nodes are depicted that were marked too small. This protects very small structures from any geometric abstraction and distortion, which would be larger for them than for larger anatomical structures.

Cut Interval Computation. The compiled zcoordinates are then used to compute cut intervals to define floors. Similar to the approach of Mindek et al. [14], floors are defined when the composition of

\begin{tabular}{rrrr}
\hline DS & DS size (voxels) & \# S & Site of Scan \\
\hline DS 1 & $512 \times 512 \times 105$ & 8 & lower thorax to pelvis \\
DS 2 & $513 \times 513 \times 108$ & 22 & head and neck area \\
DS 3 & $512 \times 512 \times 99$ & 9 & lower thorax and upper abdomen \\
\hline
\end{tabular}

Table 1: Details of the used CT data sets (DS) with their respective number of voxels, the labeled structure count (\# S), and the anatomical site of the scan (cf. Fig. 1). segmentation masks between adjacent slices changes. This results in unique floors and visually guides users to regions with high anatomical variability. Therefore, first, $L_{Z}$ is sorted in ascending order and double coordinates are removed since the method only needs knowledge about slices in which the composition changes, but not about which segmentation masks are the reason. For each remaining entry $e_{k} \in L_{Z}$ only one of the following statements (S1-S3) is correct:

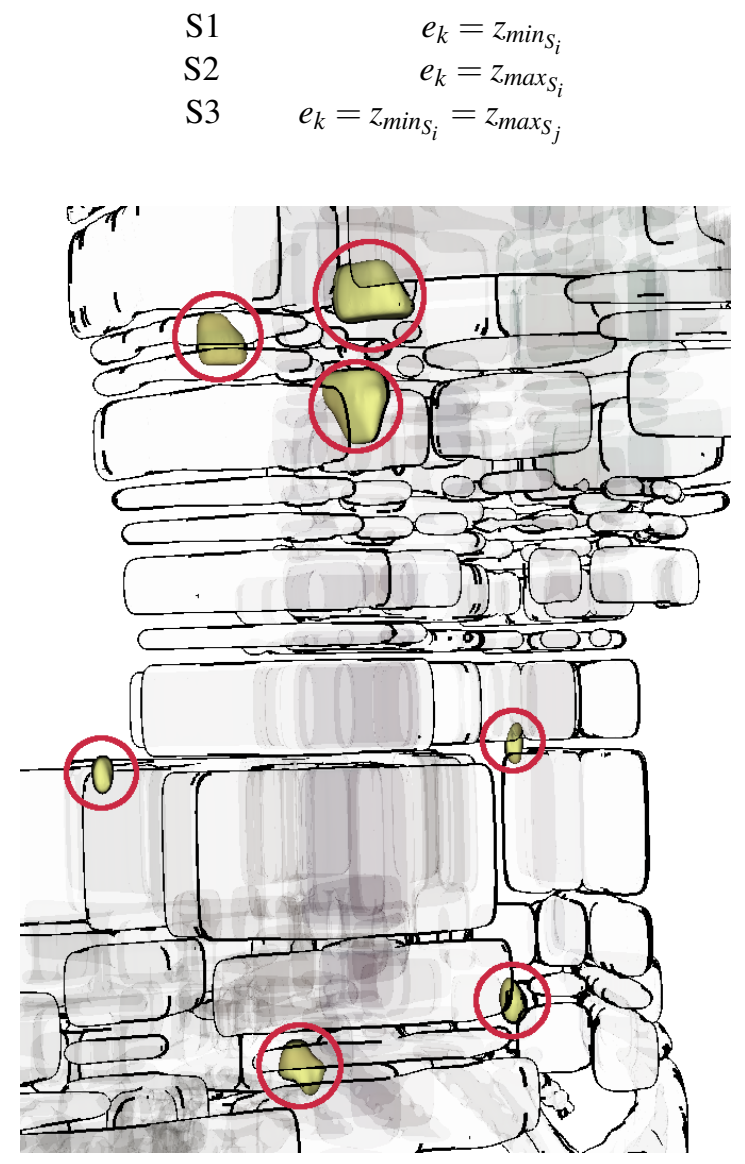

Figure 5: Lymph nodes that are flagged too small, which protects them from geometric abstraction and distortion. 
For each $e_{k} \in L_{Z}$ there exist structure segmentations $S$ that either start in slice $e_{k}(\mathrm{~S} 1)$, end in $e_{k}(\mathrm{~S} 2)$, or at least one starts while at least another ends (S3). Subsequently, all $e_{k} \in L_{Z}$ have to be flagged accordingly. Using an extended interval notation and $L_{Z}$ of Data Set 1 , the following line enables a quick understanding on how cut intervals are defined using our approach:

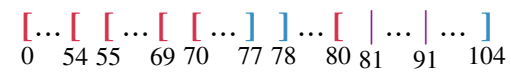

For each entry $e_{k}$, the color-coding and token show which statement is true: A red left square bracket is used for S1, a blue right square bracket is used for S2, and a violet bar is used for S3.

Subsequently, all entries are processed pairwise. Depending on which statements are true for this and the next entries $e_{k}$, the respective cut interval will be defined differently. There exist nine combinations, because, e. g., $e_{k}$ can fulfill S1 while $e_{k+1}$ can fulfill S1, S2, or S3. For example: For the slices 0 and 54 the composition of segmentation masks does not change until slice 54. Thus, the first floor will be defined from slice 0 to 53 and slice 54 will be processed in the next step. For 70 and 77, slice 77 can be included in this processing step and has to be skipped in the next step. Applying the method shown in Algorithm 1 to Data Set 1, the resulting list of cut intervals $L_{C I}$ will be:

$$
\begin{aligned}
L_{C I}=\{ & {[0,53],[54,54],[55,68],[69,69], } \\
& {[70,77],[78,78],[79,79],[80,80], } \\
& {[81,81],[82,90],[91,91],[92,104]\} }
\end{aligned}
$$

The algorithm produces the smallest number of floors with no double slices in adjacent intervals. Thus, visual distortion is minimal and the linking between original images and floor map is unique for each slice, which is important for the simultaneous exploration later. Finally, to create visual gaps between floors, empty slices are created between $L_{C I}$ intervals before mesh creation. We found that one or two slices are sufficient, because larger gaps increase the need that users have to navigate through the final visualization manually. This would be unfavorable, because medical experts are not as well used to 3D interactions as computer graphic experts.

Vertex Mesh Generation. To create meshes, the Neighboring Cells Algorithm is used [2]. However, before that, rooms have to be created inside the previously defined floors: For each floor and all segmentation masks therein, this is achieved via projection and extrusion (see Fig. 6): First, all masks are projected along the z-axis. Secondly, this shape is extruded along the floor's height. This results in rooms that are defined by the maximum axial shape of their respective structures.

This approach preserves some geometric features of the anatomical structures, which, in combination with their vertical position, supports individual recognizability. However, it also creates overlapping artifacts between rooms. This can be seen in Figure 6. Although there exists no overlap between the segmentation masks $S_{1}$ and $S_{2}$, extruding their maximum axial shape results in the overlapping rooms $R_{1}$ and $R_{2}$.

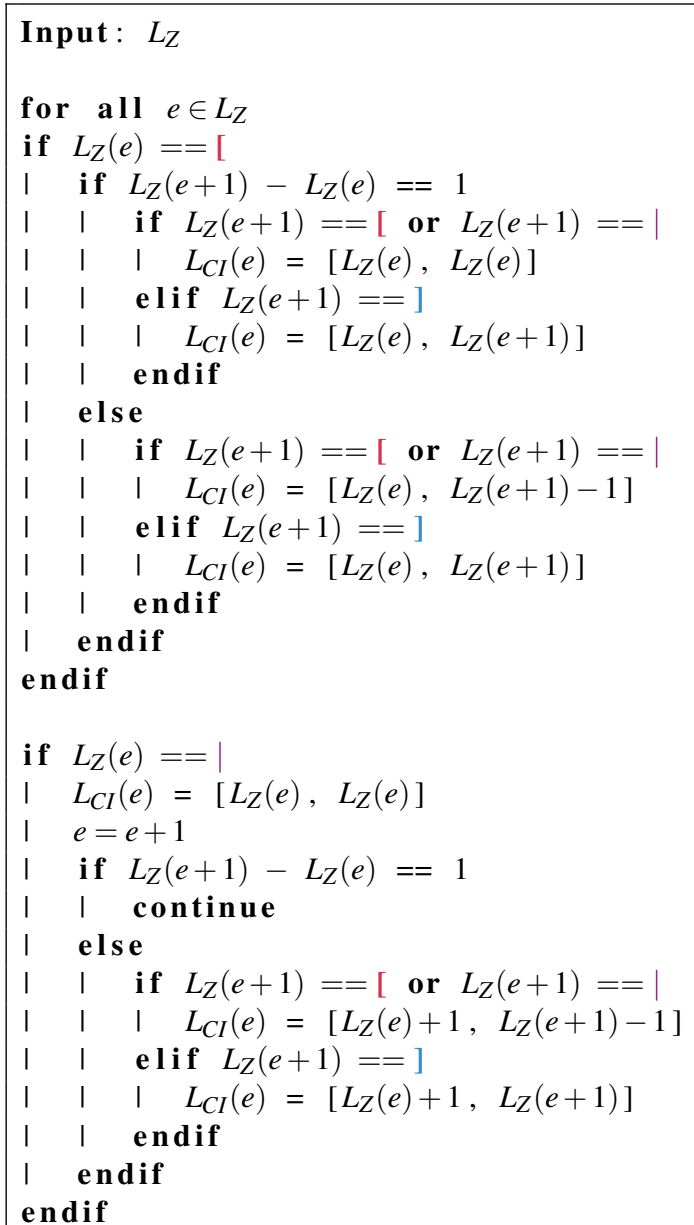

if $L_{Z}(e)==$ ]

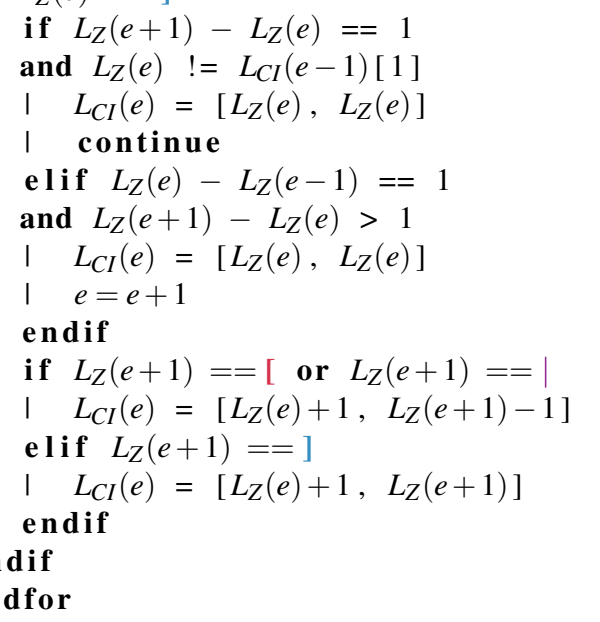

Output : $L_{C I}$

Algorithm 1: Cut Interval Generation. 
This issue is resolved by performing pairwise overlapping tests between all rooms. If overlaps exist, the overlapping volume is assigned to the smaller room. This decision was taken, because, if rooms overlap, smaller rooms are already at a higher risk to be overlooked. Thus, their visibility is increased. In Figure 6, this would be $R_{2}$ and the red-colored mediastinum, respectively.

After overlapping artifacts are resolved, processed rooms are very close to each other. Therefore, to increase visual separability of adjacent rooms, surface meshes are smoothed, e.g. via Laplace smoothing. The result is depicted in Figure 7: After overlap removal, with respect to the used color-coding, the mediastinum and lungs can be clearly distinguished. However, the visual transition

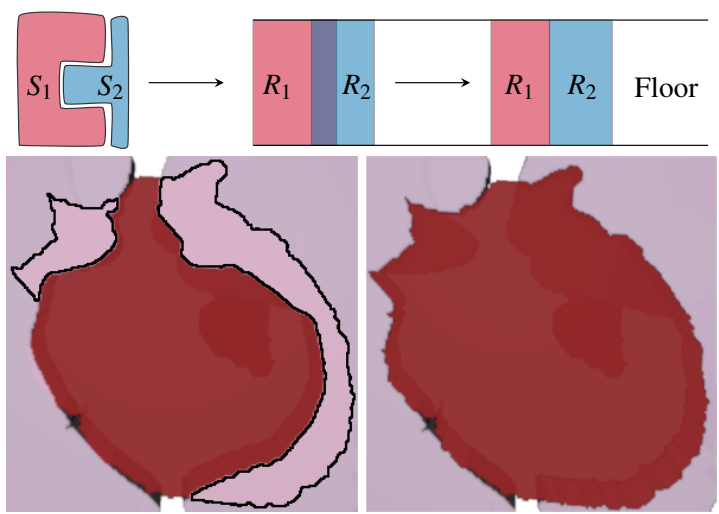

Figure 6: Top: To create rooms, the maximum axial shape of each segmentation mask $S_{i}$ is extruded along a floor. This can result in overlap artifacts between rooms $R_{i}$. This problem is addressed by assigning overlapping volume to the smaller room. Bottom: The mediastinum (red) and the lungs (rose) overlap laterally. The boundaries of the overlapping volumes are emphasized for better visibility. Since the mediastinum is smaller than both lungs, the overlapping volumes are assigned to it.

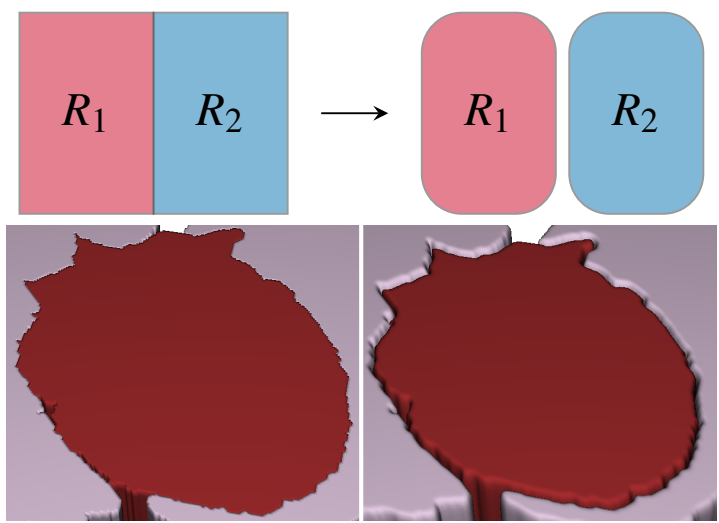

Figure 7: After extrusion and overlap removal, adjacent rooms are contiguous. Therefore, Laplacian smoothing is applied, which results in volume shrinkage. This creates gaps between adjacent meshes and valleys around the top and bottom edges. In combination with diffuse shading, rooms become more visually separated. is abrupt, which can become a problem if rooms with similar colors are too close to each other. Applying mesh smoothing creates valleys between adjacent rooms and in combination with diffuse shading, the visual separability is further increased.

Before mesh generation, users have two options to alter the transformation of individual segmentation masks into floors and rooms. First, the shape of structures can be preserved. This means that while the cut intervals in $L_{C I}$ are still used to create individual floors, not their maximum axial shape but the unaltered segmentation mask is used to create rooms. Secondly, masks can be protected from being divided into floors. To do this, a morphological dilatation operator in z-direction is used to re-merge vertical gaps that are a result of empty slices that are created between adjacent cut intervals. Both options can be combined, which is depicted in Figure 8: Here, the musculi sternocleidomastoideus are protected from divisions and geometric abstraction into rooms.

Another option that was included is that all structures in the lowest or highest floor can have their shape preserved. This can be seen in Figure 1: In the leftmost subfigure, the lowest and highest floors were processed normally. Due to their large vertical extent, they introduce a large

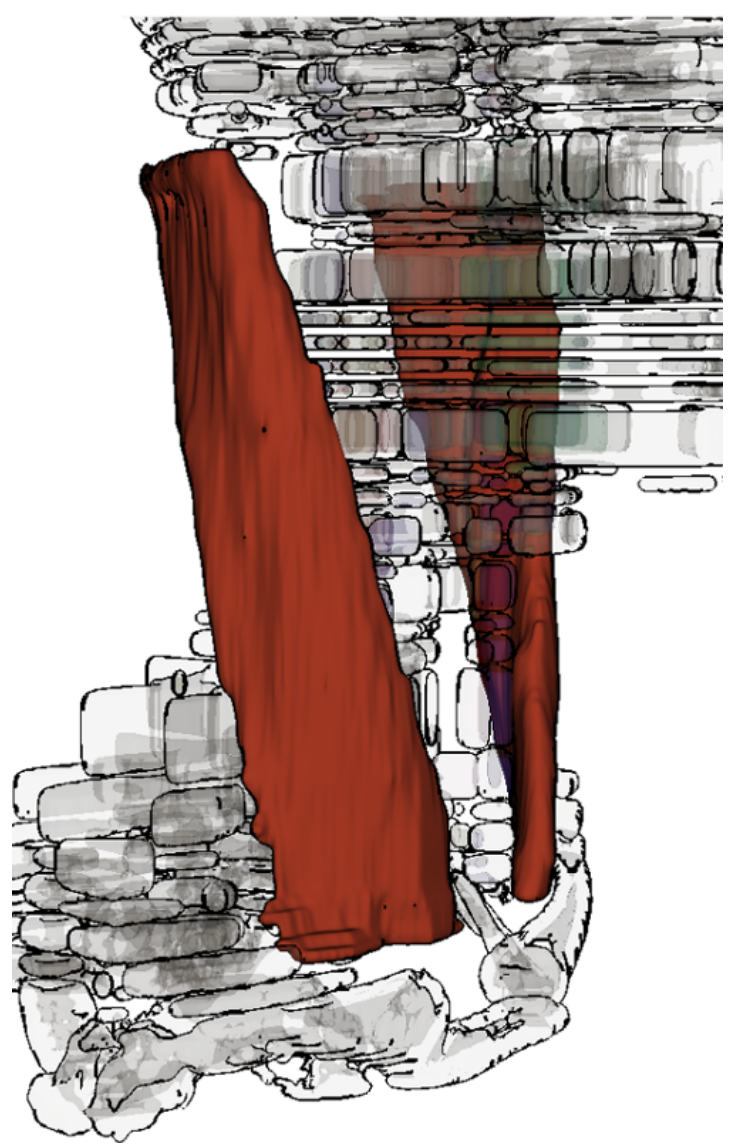

Figure 8: Users can preserve the shape of segmented structures and re-merge gaps. Here, both options were combined for the musculi sternocleidomastoideus. 

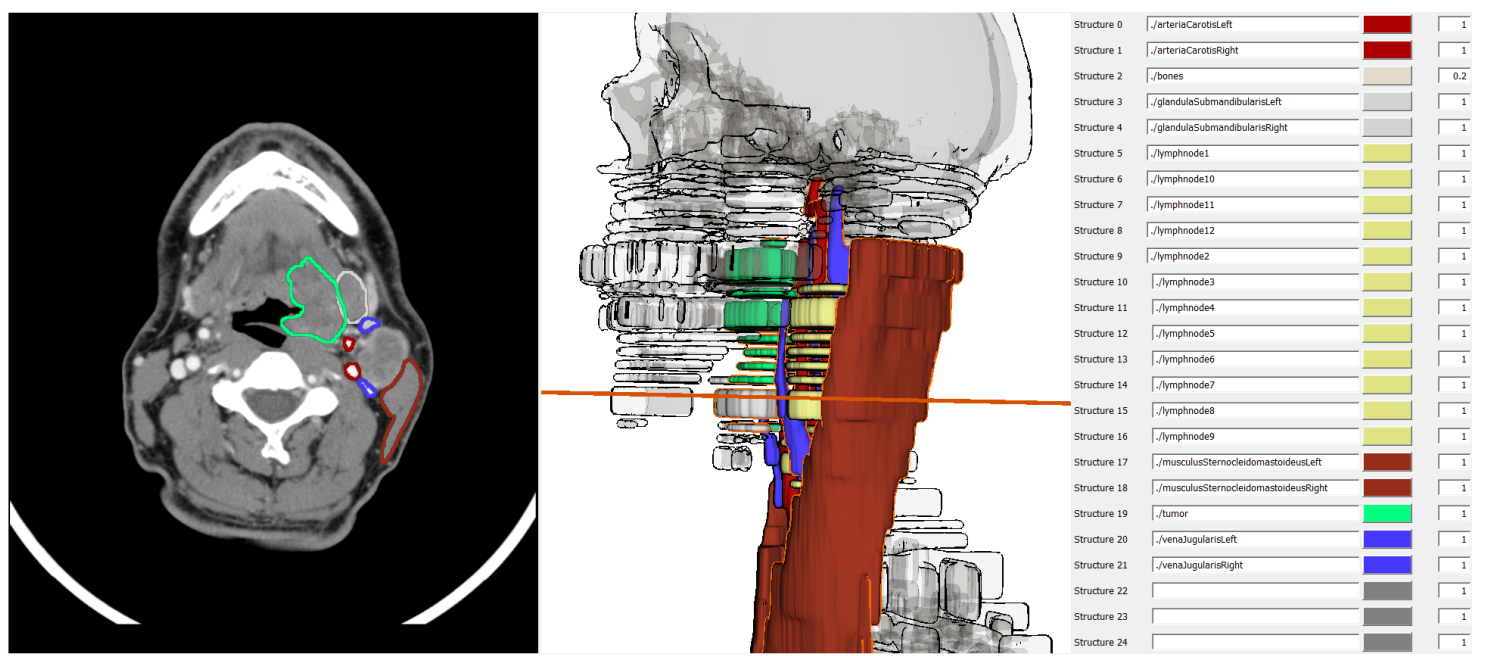

Figure 9: The GUI of the combined 2D view and floor map visualization. A tumor (green), and the left glandula submandibularis (white), carotis (red), and jugular vein (blue) are highlighted in 2D and 3D. The orange-colored frame corresponds with the currently displayed slice. On the right, the names, colors, and opacities are shown for all structures. Users can interactively adjust these options to change the floor map visualization.

geometric distortion. In contrast, for the middle subfigure, the shapes of the upper skull and collarbones were preserved, which can increase the recognizability of the depicted anatomical site considerably.

Interactive Floor Map Visualization. Figure 9 depicts the GUI that enables an interactive and simultaneous exploration of the original images and floor map. In the $2 \mathrm{D}$ view on the left, users can slice up and down through the original image stack, e. g. via mouse wheel scrolling. In the floor map, an orange-colored frame moves up and down accordingly that shows which floor the currently displayed slice belongs to. Note that this frame has to jump over gaps in the floor map, because the original image stack does not have empty slices that are virtually inserted to create individual floors. Moreover, users can select room meshes and the boundaries of the respective segmentation masks are highlighted in the 2D images. In Figure 9, various structures, such as a tumor (green), arteries (red), and veins (blue) were selected. Additionally, a geometry-based contour shading is used to highlight the $3 \mathrm{D}$ contours of selected rooms. To control occlusion

\begin{tabular}{rrrr}
\hline & \multicolumn{3}{c}{ Data Set (\# S/ \# F) } \\
Category & DS 1 (9/ 15) & DS 2 (22/ 28) & DS 3 (9/ 15) \\
\hline Z-Coordinate Extraction & 0.94 & 2.51 & 1.52 \\
\hline Cut Interval Computation & 0.01 & 0.05 & 0.01 \\
\hline Vertex Mesh Generation & & & \\
Room Extrusion & 12.66 & 32.94 & 10.93 \\
Overlap Removal & 21.68 & 47.64 & 14.34 \\
Mesh Generation & 29.65 & 64.01 & 30.92 \\
Mesh Smoothing & 2.43 & 3.08 & 4.41 \\
\hline Total Processing Time & 67.37 & 150.23 & 62.13 \\
\hline
\end{tabular}

Table 2: Computation times in seconds for each processing step from Figure 4. For each data set, the number of structures and the number of floors are given with \# S and \# F. No structure was marked too small, no shape was preserved, and no rooms were re-merged. problems, the opacities of all rooms can be adjusted on the right using order-independent transparencies [4].

\section{RESULTS}

The main result of this work is a pipeline that transforms labeled, medical image stacks into interactively explorable floor maps (see Fig. 4). Algorithm 1 is the core of the proposed method and it produces the smallest possible number of floors. Because double coordinates are removed during cut interval computation, there exist no ambiguities when the original images and the floor map are explored. In Table 2, the computation times of all processing steps are shown. They were acquired using an i5-2500 processor with $3.30 \mathrm{GHz}$.

\subsection{Evaluation}

To evaluate the proposed approach, three trained anatomists were interviewed informally and each of them filled out a questionnaire. Two interviewees are physicians (I1 and I2) and one is a biologist (I3). Similar to Figure 9, they were given a software prototype to simultaneously explore CT image stacks of three data sets (cf. Tab. 1 and Fig. 9) with either lift charts or floor maps. The interviewees were given a five-point Likert scale $(--,-, \mathrm{o},+,++)$ to answer questions. Their answers are compiled in Table 3 and are presented using a diverging red-white-blue color-scale. The questionnaires were divided into three parts, which will be explained in the following paragraphs and a discussion will be provided in the next section.

In the first part, the interviewees were asked to provide information about their clinical and technical experience. Their answers are shown in the upper part of Table 3:

Q 1 How do you rate your anatomical knowledge? 
Q 2 How familiar are you with medical visualization techniques in general?

Q 3 How familiar are you with exploration-assisting visualization techniques for medical images?

Q 4 How do you rate your spatial thinking capabilities?

For the second part, the interviewees were given instructions on how to interact with the elements of the software prototype. First, they were asked to explore the CT data sets with lift charts (cf. Fig. 2). The currently depicted slice was represented as a horizontal line in the lift chart. Subsequently, the interviewees explored the data sets with floor maps. After each exploration, they were asked to rate the exploration-assisting capabilities of each visualization technique (VT):

Q 5 How easy is it for you to get a first overview of the data set using VT?

Q 6 How easy is it for you to assess spatial relationships between anatomical structures using VT?

Q 7 How much does VT support you to find anatomical abnormalities, e.g. a tumor?

Q 8 How easy is it for you to use VT for orientation?

Q 9 How fast do you recognize segmented structures using VT?

Q 10 Overall: How much do you like using VT?

For the last part, they were asked how feasible they think each VT is for various clinical application areas.

\section{DISCUSSION}

Method Discussion. The presented pipeline requires labeled images and does not include segmentation algorithms. Depending on the data set (cf. Tab. 1), the available structure segmentations were obtained using different segmentation algorithms. For example, the liver in DS 3 was segmented using HepaVision 2 from Bourquain et al. [5], which uses a semi-automatic livewire approach. This results in a binary mask that can immediately be processed with our method. In contrast, the lymph nodes and blood vessels in DS 2 were segmented using the model-based methods of Dornheim et al. $[8,9]$ to support the treatment planning for neck dissection surgeries. These methods produce polygonal representations of segmented structures, which requires a conversion into binary masks. This is not ideal, since this conversion degrades the quality of segmentation results to some extent. However, this is not an issue, because the presented method was not developed with this use case in mind.

Although healthy anatomical structures are clearly separated from each other, obtaining segmentation masks involves some degree of uncertainty. This uncertainty is usually increased around neoplasias, e. g. tumors or metastases and, thus, segmentations can overlap. This can be seen in Figure 9, where the tumor (green) and glandula (gray) segmentations overlap. Our approach addresses these cases by assigning overlapping volume to the smaller structure. However, there exist cases where structures are embedded in each other, e. g. tumors or metastases in organs. As long as the neoplastic structures are smaller in volume than the surrounding organ tissue, overlapping volume will be assigned to the neoplasm. Thus, tumors and metastases will not become occult in the final floor map. In this scenario, the overlapping and neoplasm room volumes are identical. However, if the embedded, cancerous tissue is larger than the remaining healthy tissue, the overlapping volume would be wrongfully labeled healthy and, thus, the cancerous tissue will be completely omitted. Such problematic cases could be addressed by enabling users to label embedded structures favored. As a result, overlapping volume would always be assigned to the favored structure, although it has the larger volume. Moreover, the proposed approach does not distinguish between elongated and spherical structures. Therefore, blood vessels can be heavily distorted if their shape is not preserved (cf. Figure 8).

Result Performance Discussion. The room extrusion, overlap removal, and mesh generation steps require the most processing time. The performance of each step could be improved via parallel processing of structures.

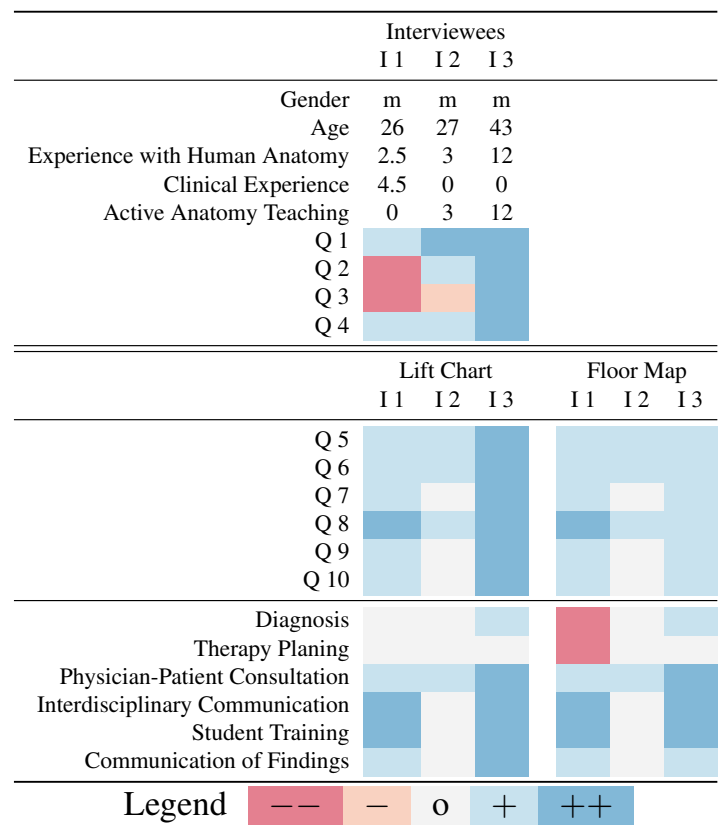

Table 3: Evaluation results of the informal interview and questionnaires. The upper part shows how the interviewees rate their anatomical and technical knowledge. Numbers mean in years. The lower parts show their assessments about the exploration-supporting capability and clinical versatility of each visualization technique. 
In case of the overlap removal step, a well-elaborated and implemented process and resource management would be required, because, using the presented approach, extruded structures are processed pairwise. Regarding the mesh generation, the measured times include the transformation of all structures into meshes. Thus, for example, the average processing time per structure for Data Set 2 was 2.91 seconds. To increase the performance of this step, the cell extent of the Neighboring Cells Algorithm can be increased [2], which can decrease the mesh quality considerably. However, the processing into surface meshes that are used for the floor map visualization has only to be done once.

Evaluation Results Discussion. The interviewees were encouraged to think aloud while exploring the original images with lift charts and floor maps. This revealed two limitations of our approach: First, although Algorithm 1 produces the smallest possible number of floors, the interviewees still reported that the final presentation would include too much visual clutter (Quote: "There is too much going on."). This is depicted in Figure 9 that shows a heavily dissected mandible. From a cognition perspective, this can be explained with the work of Alvarez and Cavanagh [1]: For orientation and navigation tasks, we process and store visuo-spatial stimuli and construct a so-called mental map and they are our mental representation of our spatial environment. However, the required resource to do so, namely the Visuo-Spatial Working Memory, is limited and heavily depends on the visual complexity and number of displayed objects. Whereas an anatomical structure is represented by one bar in a lift chart, it can be represented by multiple rooms in a floor map. Therefore, although the geometric appearance of rooms can be considered simple, displaying them all appears to be overwhelming. This limitation could be addressed by including knowledge about the in-plane anatomical variability so that adjacent rooms in regions with a low variability get merged.

Secondly, Algorithm 1 divides image stacks into floors when the composition of segmentation masks changes between adjacent slices. This was done to guide the attention of users towards regions with a large anatomical variability. Although this approach is used in other visualization domains [14], the interviewees remarked that they are not used to this type of orientation and that the floor map "requires a lot of reading". They use certain anatomical landmarks for orientation, e.g. the vertebrae for the upper body. In regard of the lift charts of Data Set 1 and 3, the interviewees noted that horizontal divisions in the bar that represents the spine and textual descriptions, namely C1-C7 for cervical, T1-T12 for thoracic, and L1-L5 for lumbar vertebrae, would be beneficial to further increase exploration support. This is related to the method of Balabanian et al. [3], who extended lift charts to be applicable for hierarchical relationships between anatomical structures. Therefore, the proposed floor division approach should also be reviewed with respect to anatomical landmarks.

In addition, the evaluation showed the clinical feasibility of the proposed approach (cf. Tab. 3). For diagnosis and therapy planning, e.g. in cases of cancer or to select positions for multiple radiation sources for radiation therapies, distances between anatomical structures are an important decision criterion. Although our approach introduces less geometric abstraction than other methods, by using the maximum axial shape of anatomical structures to create rooms in-plane distances become heavily distorted. For communication tasks, i. e. with patients, students, and colleagues, our approach was rated just as good as lift charts. However, when the aforementioned limitations are addressed, the overall feasibility of the presented approach should improve.

\section{CONCLUSION \& FUTURE WORK}

In this paper, a novel visualization approach was presented that transforms labeled medical image stacks into a three-dimensional map layout, namely floor maps. Furthermore, it was discussed how the resulting visualizations can be combined with the conventional slicebased exploration of CT image stacks. The proposed approach was evaluated by interviewing three anatomy experts, which revealed two shortcomings: First, the main goal was to offer exploration support for medical image stacks via maps. However, although the presented method guarantees to minimize the number of produced floors and rooms, it was reported that anatomical structures are still represented by too many visual entities [1]. This shortcoming could be addressed by generating good default views and limiting the number of presented rooms, for example, by pulling out corresponding floors like drawers during a slice-based exploration $[11,18]$. The exploration-supporting facilities of floor maps should not be hindered, because humans are still able to construct mental maps from piecewise, sequentially presented maps for orientation and navigation tasks [21]. Secondly, a landmark-based approach to construct floors and rooms appears to be beneficial. However, using vertebrae as landmarks only works for the upper body. Therefore, the method has to be adjusted with respect to the anatomical area that was scanned.

Currently, the approach is limited to visualize one data set at a time. A potentially useful extension is the integration of multiple data sets of different modalities. Inspired by Ropinski et al. [16], a combination of CT and Positron Emission Tomography (PET) scans could be interesting: While morphological information from CT scans would still be used to create floors and rooms that give a spatial context, physiological information from PET scans could be used to emulate certain functionalities inside rooms, for example light sources. Rooms that represent segmented structures with an increased PET 
activity, e.g. tumors or metastases, could be presented in a lights on mode, while all other rooms are presented normally in a lights out mode. We argue that this feature would strongly guide the attention of users towards interesting (malignant) structures.

Finally, we think about applying varying degrees of geometric abstraction to different types of anatomy, i. e. organs and vessel trees [19]. For organs, instead of extruding their maximum axial shape, their axis or objectaligned bounding boxes could be used in floor map layouts. Methods, such as the Douglas-Peucker algorithm [10], could be used to simplify vessel trees, since they are geometrically similar to rivers and estuaries.

\section{ACKNOWLEDGMENTS}

This work is partly funded by the Federal Ministry of Education and Research within the Forschungscampus STIMULATE (13GW0095A). We want to thank Wolfgang D'Hanis, Sven Schumann, and Leonardo Nardi for being our interviewees and providing us with feedback.

\section{REFERENCES}

[1] G. Alvarez and P. Cavanagh. The Capacity of Visual Short-Term Memory is Set Both by Visual Information Load and by Number of Objects. Psychol Sci, 15(2):106-111, 2004.

[2] R. Bade, O. Konrad, and B. Preim. Reducing Artifacts in Surface Meshes Extracted from Binary Volumes. Journal of WCSG, 15:67-74, 2007.

[3] J.-P. Balabanian, I. Viola, and E. Gröller. Interactive Illustrative Visualization of Hierarchical Volume Data. In Proc. of Graphics Interface, pages 137-144, 2010.

[4] P. Barta, B. Kovács, L. Szecsi, and L. SzirmayKalos. Order Independent Transparency with PerPixel Linked Lists. In Proc. of CESCG, pages 51-57, 2011.

[5] H. Bourquain, A. Schenk, F. Link, B. Preim, G. Prause, and H.-O. Peitgen. HepaVision2 - A Software Assistant for Preoperative Planning in Living-Related Liver Transplantation and Oncologic Liver Surgery. In Proc. of CARS, pages 341346, 2002.

[6] L.-C. Chen, C.-H. Wu, T.-S. Shen, and C.-C. Chou. The Application of Geometric Network Models and Building Information Models in Geospatial Environments for Fire-Fighting Simulations. Comput Environ Urban, 45:1-12, 2014.

[7] S. Diepenbrock, J.-S. Praßni, F. Lindemann, H.-W. Bothe, and T. Ropinski. Interactive Visualization Techniques for Neurosurgery Planning. In Proc. of. Eurographics, pages 13-16, 2011.
[8] J. Dornheim, D. Lehmann, L. Dornheim, B. Preim, and G. Strauss. Reconstruction of Blood Vessels from Neck CT Datasets using Stable 3D MassSpring Models. In Proc. of Eurographics Workshop on VCBM, pages 77-82, 2008.

[9] J. Dornheim, H. Seim, B. Preim, I. Hertel, and G. Strauss. Segmentation of Neck Lymph Nodes in CT Datasets with Stable 3D Mass-Spring Models. In Proc. of MICCAI, volume 4191 of LNCS, pages 904-911, 2006.

[10] D. H. Douglas and T. K. Peucker. Algorithms for the Reduction of the Number of Points Required to Represent a Digitized Line or its Caricature. Cartographica, 10(2):112-122, 1973.

[11] M. Gai and G. Wang. Indoor3D: A WebGL Based Open Source Framework for 3D Indoor Maps Visualization. In Proc. of Web3D, pages 181-187, 2015.

[12] M. Goetz and A. Zipf. Extending OpenStreetMap to Indoor Environments: Bringing Volunteered Geographic Information to the Next Level. In Proc. of UDMS, volume 2011, pages 47-58, 2011.

[13] B. Hagedorn, M. Trapp, T. Glander, and J. Döllner. Towards an Indoor Level-of-Detail Model for Route Visualization. In Proc. of MDM, pages 692-697, 2009.

[14] P. Mindek, G. Mistelbauer, M. E. Gröller, and S. Bruckner. Data-Sensitive Visual Navigation. Comput Graph, 67:77-85, 2017.

[15] F. Ritter, T. Boskamp, A. Homeyer, H. Laue, M. Schwier, F. Link, and H.-O. Peitgen. Medical Image Analysis. IEEE Pulse, 2(6):60-70, 2011.

[16] T. Ropinski, S. Hermann, R. Reich, M. Schäfers, and K. Hinrichs. Multimodal Vessel Visualization of Mouse Aorta PET/CT Scans. IEEE T Vis Comput Gr, 15(6):1515-1522, 2009.

[17] C. Tietjen, B. Meyer, S. Schlechtweg, B. Preim, I. Hertel, and G. Strauss. Enhancing Slice-based Visuaizations of Medical Volume Data. In Proc. of Eurographics/IEEE-VGTC Symposium on Visualization, pages 123-130, 2006.

[18] P.-P. Vázquez, M. Feixas, M. Sbert, and W. Heidrich. Viewpoint Selection Using Viewpoint Entropy. In Proc. of VMV, pages 273-280, 2001.

[19] I. Viola and T. Isenberg. Pondering the Concept of Abstraction in (Illustrative) Visualization. IEEE T Vis Comput Gr, 24(9):2573-2588, 2018.

[20] X. Yin, P. Wonka, and A. Razdan. Generating 3D Building Models from Architectural Drawings: A Survey. IEEE Comput Graph, 29(1):20-30, 2009.

[21] H. D. Zimmer. The Construction of Mental Maps Based on a Fragmentary View of Physical Maps. J Educ Psychol, 96(3):603-610, 2004. 